\title{
Mass and Lifetime Measurements with Exclusive B Reconstruction at CDF
}

\author{
Jonathan D. Lewis \\ The CDF Collaboration \\ Fermi National Accelerator Laboratory \\ P.O. Box 500, Batavia, Illinois 60510
}

July 1994

Lecture given at the 1994 Recontre de Physique de la Vallee d'Aoste on Results and Perspectives in Particle Physics, La Thuile, Italy, March 6-12, 1994. 


\section{Disclaimer}

This report was prepared as an account of work sponsored by an agency of the United States Government. Neither the United States Government nor any agency thereof, nor any of their employees, makes any warranty, express or implied, or assumes any legal liability or responsibility for the accuracy, completeness, or usefulness of any information, apparatus, product, or process disclosed, or represents that its use would not infringe privately owned rights. Reference herein to any specific commercial product, process, or service by trade name, trademark, manufacturer, or otherwise, does not necessarily constitute or imply its endorsement, recommendation, or favoring by the United States Government or any agency therenf: The views and opinions of authors expressed herein do not necessarily state or reflect those of the United States Government or any agency thereof. 
CDF/PUB/BOTTOM/PUBLIC/2603

FERMILAB-CONF-94/128-E

July 7,1994

\title{
MASS AND LIFETIME MEASUREMENTS WITH EXCLUSIVE $B$ RECONSTRUCTION AT CDF
}

\author{
Jonathan D. Lewis \\ Fermi National Accelerator Laboratory \\ P.O. Box 500 \\ Batavia, IL 60510 \\ for the CDF Collaboration
}

\begin{abstract}
Lifetimes and masses of $B$ mesons have been measured in exclusively reconstructed decays including $J / \psi \rightarrow \mu^{+} \mu^{-}$decays. In $19.3 \mathrm{pb}^{-1}$ of $\bar{p} p$ collisions at $\sqrt{s}=1.8 \mathrm{TeV}$ taken in 1992-93 at the Fermilab Tevatron collider, approximately $300 \mathrm{~B}$ mesons have been fully reconstructed. The measurements require precise determination of the helical track parameters of charged particle tracks. We find $M\left(B_{S}^{0}\right)=5367.7 \pm 2.4 \pm 4.8 \mathrm{MeV} / c^{2}$, $\tau\left(B^{+}\right)=1.61 \pm 0.16 \pm 0.05 \mathrm{ps}$, and $\tau\left(B^{0}\right)=1.57 \pm 0.18 \pm 0.08 \mathrm{ps}$.
\end{abstract}

Lecture given at the 1994 Rencontre de Physique de la Vallée d'Aoste on Results and Perspectives in Particle Physics, La Thuile, Italy, 6-12 March 1994. 


\section{Introduction}

In the 1988-89 Tevatron collider run, CDF showed the capability to trigger on and to cleanly reconstruct $B \rightarrow J / \psi \mathrm{X}$ decays where $J / \psi \rightarrow \mu^{+} \mu^{-}$in $\bar{p} p$ collisions at $\sqrt{s}=1.8 \mathrm{TeV}$ ) The addition of a Silicon Vertex Detector (SVX) and increased dimuon acceptance have paved the way for CDF to make high-statistics $B$ physics measurements beyond production studies. In 1993, CDF published a $B_{S}^{0}$ mass measurement ${ }^{2)}$ of $5383.3 \pm 4.5 \pm 5.0 \mathrm{MeV} / c^{2}$ and an average $B$ lifetime ${ }^{3)}$ of $1.46 \pm 0.06 \pm 0.06 \mathrm{ps}$. We have included the full $19.3 \mathrm{pb}^{-1}$ from the $1992-1993$ running of the Tevatron ("Run 1A") and used improved charged particle track reconstruction and tracking chamber alignments to improve the $B_{S}^{0}$ mass measurement and to measure the separate lifetimes of charged and neutral $B$ mesons.

The CDF detector is described in detail elsewhere.) We describe briefly below the detector subsystems crucial to the reconstruction of $B$ decays: the SVX, the Central Tracking Chamber (CTC), the muon detectors, and the dimuon trigger. The SVX is a four-layer silicon microstrip detector. The strips run parallel to the beam axis $(z)$ and have a pitch of $60 \mu \mathrm{m}$ in the inner three layers and $55 \mu \mathrm{m}$ in the outermost layer. The detector covers the range $z<30 \mathrm{~cm}$, where $z$ is measured from the center of the detector. The spatial resolution of the device is $13 \mu \mathrm{m}$ with an impact parameter resolution of $\left(13+40 / p_{T}\right) \mu \mathrm{m}$, where $p_{T}$ is the component of a particle's momentum transverse to the beam axis measured in $\mathrm{GeV} / c$. The transverse profile of the beam is approximately circular with an rms width of $40 \mu \mathrm{m}$. The longitudinal rms length of the luminous region within the CDF detector is about $30 \mathrm{~cm}$. The $\mathrm{CTC}^{5)}$ is an 84-layer open-cell drift chamber. It contains five 12-layer superlayers of axial wires and four interleaved 6-layer superlayers with $\pm 3^{\circ}$ stereo angle. The momentum resolution of the combined CTC-SVX system is $\delta p_{T} / p_{T}=\sqrt{\left(0.0009 p_{T}\right)^{2}+(0.0066)^{2}}$, where $p_{T}$ is measured in $\mathrm{GeV} / c$.

Two sets of detectors are used to identify low-momentum muons. The Central Muon system (CMU) includes four layers of drift chambers forming a cylinder outside the central calorimeter. The calorimeter includes about 4 interaction lengths of material. The Central Muon eXtension (CMX) is an 8-layer set of drift chamber tubes that form the frustrum of a cone centered on the beam axis and follows approximately 5 interaction lengths of material. The CMU covers the pseudorapidity range $|\eta|<0.6$ and $85 \%$ of azimuth, and the CMX covers $0.6<|\eta|<1.0$ and $67 \%$ of azimuth, where $\eta \equiv-\ln \left(\tan \frac{\theta}{2}\right)$ and $\theta$ is the polar angle relative to the proton beam. Identification of muons requires matching charged particle tracks in the CTC to track stubs reconstructed in the muon drift tubes. The resolution including the effects of multiple Coulomb scattering along the azimuthal direction is $\delta x=15 \mathrm{~cm} / p_{T}$ for CMU and $36 \mathrm{~cm} / p_{T}$ for CMX and along the longitudinal direction is $\delta z=18 \mathrm{~cm} / p_{T}$ for CMU, where $p_{T}$ is measured in $\mathrm{GeV} / c$.

CDF employs a three level triggering scheme. Muons are identified at the first level by a coincidence between radially aligned wires in the muon detectors. The temporal separation of the hits in the two layers is required to be consistent with a muon of $p_{T}>3 \mathrm{GeV} / \mathrm{c}$. If the first level of trigger is satisfied, detector input is disabled while the second level of the trigger evaluates the event. Dimuon events are require to have at least one of the first level coincidences matched to a track with $p_{T}>3 \mathrm{GeV} / c$ found by the CFT online track processor.) Events that pass the second level requirements are digitized and passed to a CPU farm that performs a software reconstruction analysis. Events that contain at least two reconstructed muon candidates are included in a dimuon data sample for further analysis. In the $B$ mass and 
lifetime measurements, events are required to have two opposite charge muon candidates with the track projecting to a track "stub" in the muon chambers within 4 standard deviations of the prediction in the azimuthal view, and in the CMU system the same requirement is made in the $z$ view as well. Other event selection criteria are described separately for the two measurements.

\section{$2 \quad B$ Meson Masses}

$B$ mesons are of special interest because they contain a heavy quark and a light quark. Models of the dynamics of $B$ mesons can treat the $b$ quark non-relativistically, while the light quark is relativistic. Theoretical calcultaions of $B$-meson decay widths are simpified because for the most part the light quark can be treated as a spectator to the heavy quark decay. Measurement of $B$ masses can help determine the QCD binding potential. Recent predictions ${ }^{7)}$ of the $B_{S}^{0}$ mass are in the range 5345 to $5390 \mathrm{MeV} / \mathrm{c}^{2}$.

We reconstruct $B^{+} \rightarrow J / \psi K^{+}, B^{0} \rightarrow J / \psi K^{* 0}$, and $B_{S}^{0} \rightarrow J / \psi \phi$ candidates* with $J / \psi \rightarrow \mu^{+} \mu^{-}$, $K^{* 0} \rightarrow K^{+} \pi^{-}$, and $\phi \rightarrow K^{+} K^{-} . J / \psi$ candidates are formed from pairs of oppositely charged muons that satisfy the matching requirement within 3 standard deviations using the full offline reconstruction analysis. The two tracks are constrained to originate from a common vertex, and we require the dimuon mass to be within $100 \mathrm{MeV} / c^{2}$ of the world average $J / \psi$ mass. ${ }^{8)}$ For each mode considered, any charged track other than those used to form the muon pair can be considered as a pion, and as a kaon if $p_{T}>2 \mathrm{GeV} / c$. $K^{* 0}$ candidates are formed from pairs of charged particle tracks that have a combined mass within $100 \mathrm{MeV} / \mathrm{c}^{2}$ of $896 \mathrm{MeV} / \mathrm{c}^{2}$ where one track is assigned the kaon mass and the other the pion mass. If both particle assignments satisfy this requirement, the choice with mass closest to $896 \mathrm{MeV} / \mathrm{c}^{2}$ is used. $\phi$ candidates are formed from pairs of tracks assigned the kaon mass with combined mass within $10 \mathrm{MeV} / \mathrm{c}^{2}$ of $1019 \mathrm{MeV} / \mathrm{c}^{2}$. The momentum each track is corrected for multiple scattering and $d E / d x$ energy loss. SVX information is used for tracks if the $\chi^{2}$ per degree of freedom is less than 6 for the extrapolation for the CTC track to at least 3 SVX layers.

The mass of the three- or four-track system is calculated subject to the constraints that the tracks originate from a common decay vertex and the parent momentum points to beam position with $C L\left(\chi^{2}\right)>1 \%$. The beam position is calculated for each store from a fit of minimum-bias events. The following additional cuts are imposed:

\begin{tabular}{|l|c|c|c|c|}
\hline Mode & $\begin{array}{c}p_{T}(B) \\
(\mathrm{GeV} / c)\end{array}$ & $\begin{array}{c}p_{T}\left(K, K^{* 0}, \phi\right) \\
(\mathrm{GeV} / c)\end{array}$ & $\begin{array}{c}c \tau \\
(\mu \mathrm{m})\end{array}$ & $\begin{array}{c}\left|\Delta M\left(K^{*} / \phi\right)\right| \\
\left(\mathrm{MeV} / c^{2}\right)\end{array}$ \\
\hline$J / \psi K^{+}$ & $>8$ & $>2$ & $>100$ & - \\
$J / \psi K^{* 0}$ & $>8$ & $>3$ & $>100$ & $<50$ \\
$J / \psi \phi$ & $>6$ & $>2$ & $>0$ & $<10$ \\
\hline
\end{tabular}

The cut on proper decay length (cr) is applied for all events even if none of the tracks is reconstructed in the SVX. A binned likelihood fit to a Gaussian signal and linear background function is performed for each of the modes studied. The width of the Gaussian is allowed to float in the fit. The results of the fits are listed in table 1, including the number of events under the peak, the most probable mass, the width of the Gaussian, and the background level per

"Unless otherwise stated, charge-conjugate decays are included in the results. 
Table 1: Results for fits for $B^{+}, B^{\circ}$ and $B_{S}^{0}$ masses.

\begin{tabular}{|c|c|c|c|c|}
\hline Meson & Number & $\begin{array}{c}\text { Mass } \\
\left(\mathrm{MeV} / c^{2}\right)\end{array}$ & $\begin{array}{c}\text { Width } \\
\left(\mathrm{MeV} / c^{2}\right)\end{array}$ & $\begin{array}{c}\text { Background } \\
\left(\text { per } 5 \mathrm{MeV} / c^{2}\right)\end{array}$ \\
\hline$B^{+}$ & $140 \pm 15$ & $5279.6 \pm 1.7$ & $15.2 \pm 1.8$ & $3.2 \pm 0.3$ \\
$B^{0}$ & $57 \pm 10$ & $5279.9 \pm 2.5$ & $13.6 \pm 2.2$ & $1.7 \pm 0.2$ \\
$B_{S}^{0}$ & $33 \pm 7$ & $5367.7 \pm 2.4$ & $10.5 \pm 1.9$ & $1.1 \pm 0.1$ \\
\hline
\end{tabular}

$5 \mathrm{MeV} / c^{2}$. The uncertainties shown are statistical only. The mass distributions with the fit results superimposed are shown in figures 1,2 , and 3 .

We have studied several possible sources of systematic uncertainties. Each of several parameters is varied within its error, and event-by-event mass shifts are computed. The average shift is used as an uncertainty estimate.

- $p_{T}$ Scale

The strength of the magnetic field is checked to see that we reconstruct $\psi$ and $\Upsilon$ with the world-average masses. Mass shifts are computed with the field strength varied by its uncertainty.

- Material Scale

We determine the absolute scale for $d E / d x$ and multiple scattering corrections from the radiative tails in the $E / P$ distribution for high- $p_{T}$ electrons from $Z^{0}$ decays. Mass shifts are computed with scale varied within its measured error.

- Mass Fitting

We vary the binning of the histogram used in the fit, use an unbinned fit, and fit with the resolution fixed to various values.

- Pointing

We calculate mass shifts without the constraint that the $B$ come from the average beam position.

- Track Fit Covariance Matrix Scale

We scale elements of the covariance matrix up and down to reflect uncertainty in the tracking errors. The average event-by-event mass shift is taken to be the uncertainty.

- Selection Criteria

We vary the momentum, decay-length and daughter-mass cuts to check for a bias. We fit the $B$-mass distributions for many sets of cuts. In order not to count the statistical uncertainty twice, for a maximum mass change $\delta$ and a statistical uncertainty $\sigma$ from the standard fit, we define the uncertainty due to selection criteria $\sigma_{c}=\sqrt{\delta^{2}-\sigma^{2}}$.

- False Curvature

Residual alignment errors can introduce a false component to the curvature of reconstructed tracks. We observe that the $J / \psi$ mass can vary within a $5 \mathrm{MeV} / c^{2}$ range as a function of the $\mu^{+}$and $\mu^{-}$momenta. Also, we observe a $4.8 \mathrm{MeV} / c^{2}$ difference between the fit masses of $B^{+}$and $B^{-}$. An ad hoc additive correction smooths the $J / \psi$ mass as 


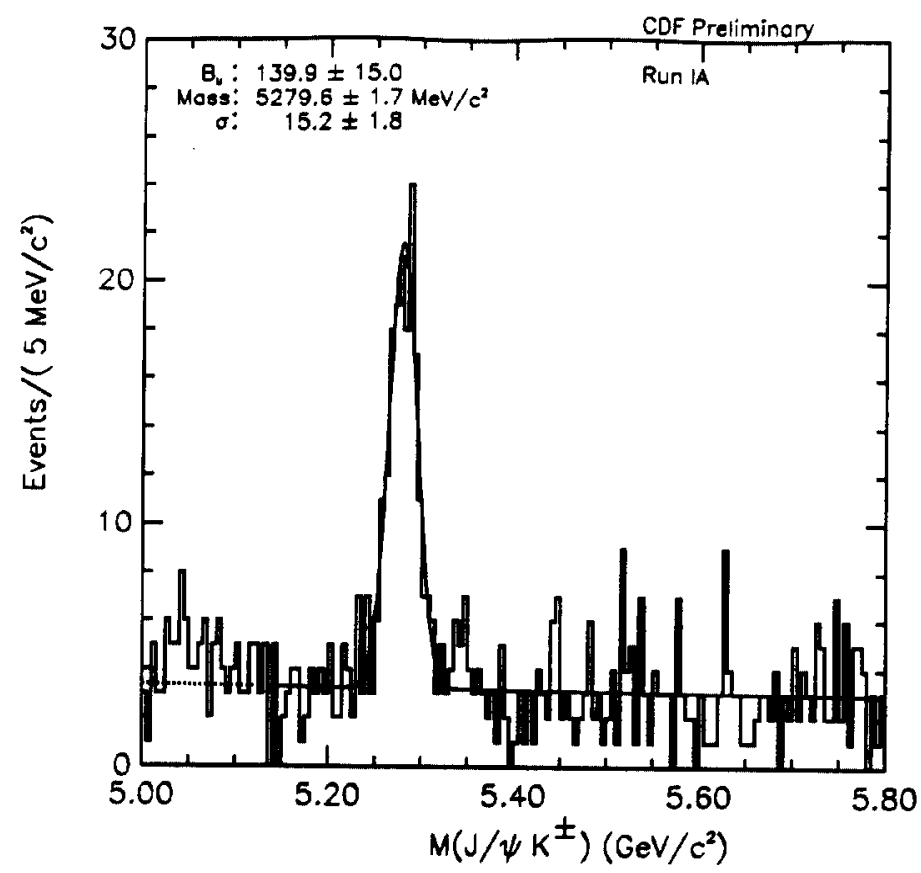

Figure 1: Mass distribution for $B^{+} \rightarrow J / \psi K^{+}$candidates. The solid line indicates the result of the fit.

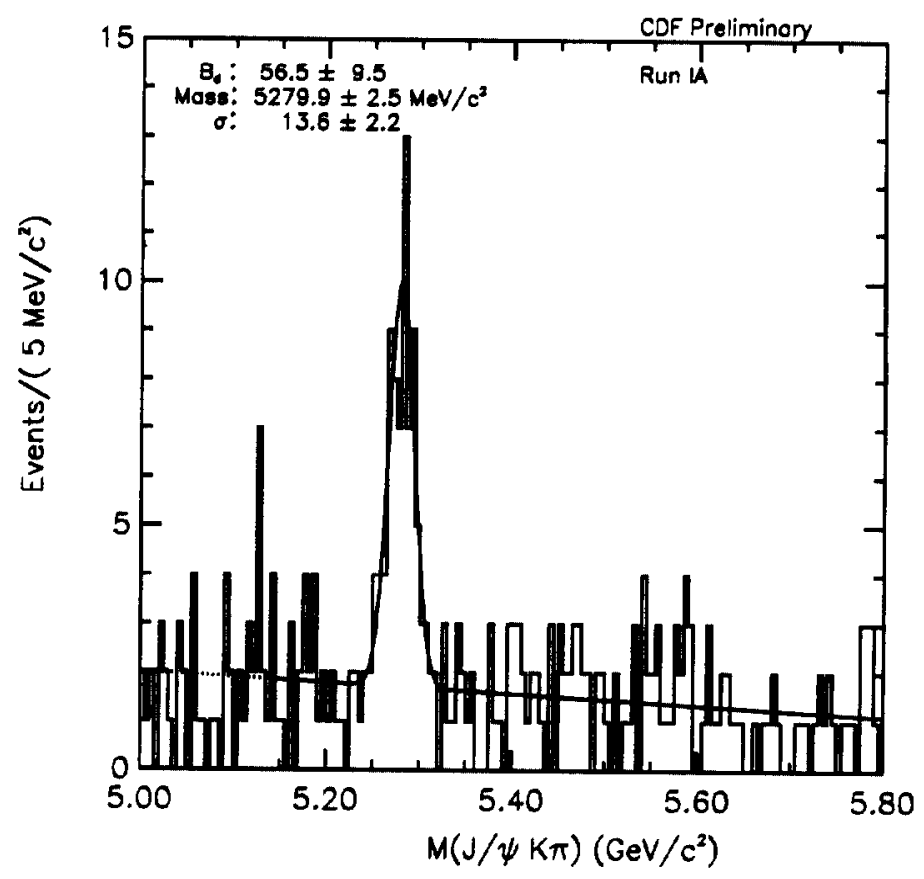

Figure 2: Mass distribution for $B^{0} \rightarrow J / \psi K^{*}$ candidates. The solid line indicates the result of the fit. 


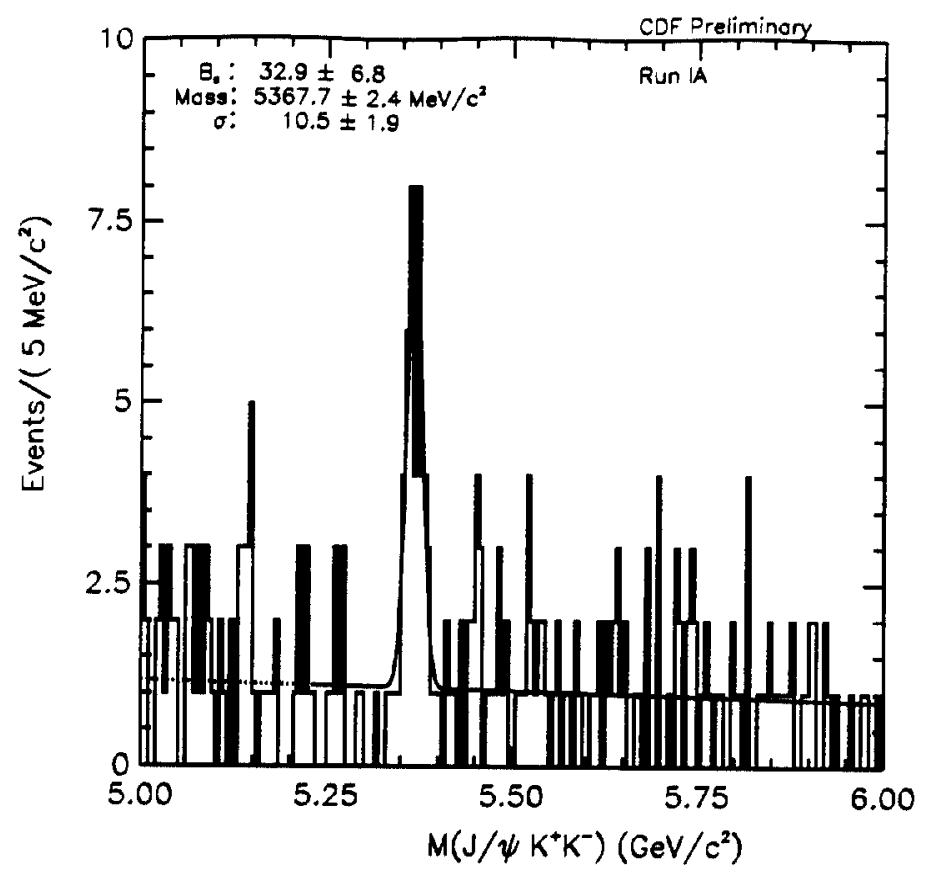

Figure 3: Mass distribution for $B_{a}^{0} \rightarrow J / \psi \phi$ candidates. The solid line indicates the result of the fit.

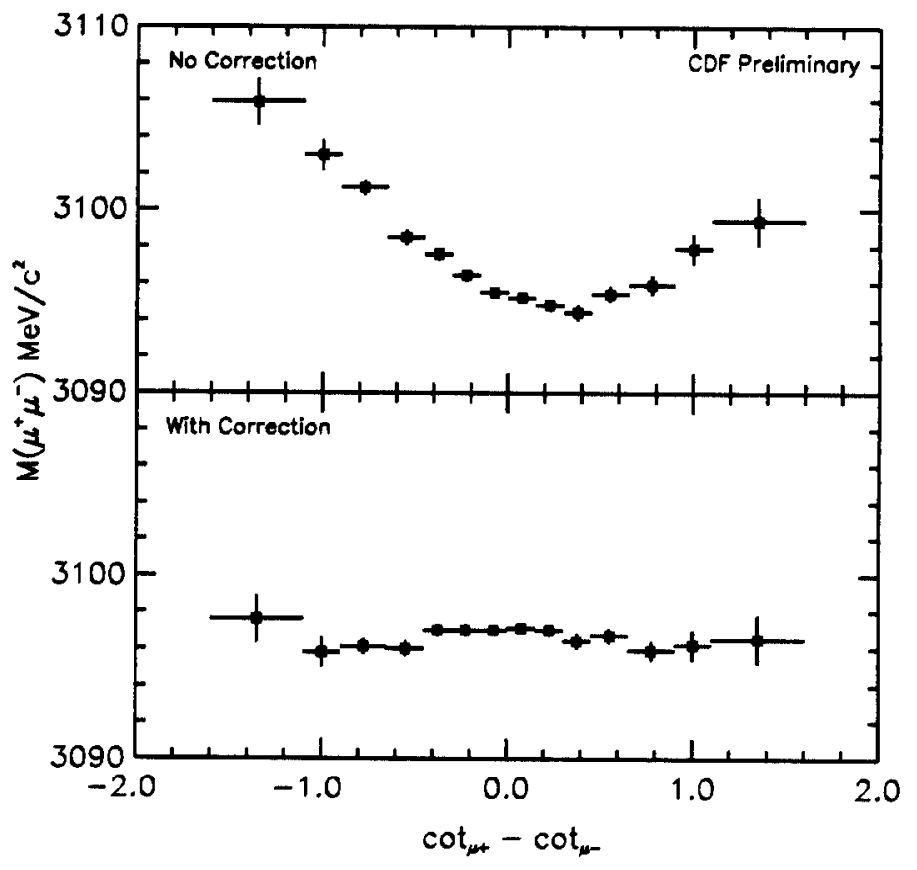

Figure 4: Mean $J / \psi$ mass as a function of polar opening angle between the two muons (upper), and the same distribution after applying an ad hoc correction to the measured muon-track polar angles (lower). 
Table 2: Fit $B$ mass for various polar opening angle cuts.

\begin{tabular}{|c|c|c|c|c||c|c|c|c|}
\hline Meson & $\begin{array}{c}\Delta \cot \theta<x \\
(J / \psi, X)\end{array}$ & $\begin{array}{c}\Delta \cot \theta>x \\
(J / \psi, X)\end{array}$ & $x$ & syst & $\begin{array}{c}\Delta \cot <x \\
(K / \pi, K)\end{array}$ & $\begin{array}{c}\Delta \cot >x \\
(K / \pi, K)\end{array}$ & $x$ & syst \\
\hline$B^{+}$ & 5278.2 & 5280.2 & 0.35 & 1.0 & - & - & - & - \\
$B^{0}$ & 5280.0 & 5280.4 & 0.30 & 0.2 & 5277.4 & 5282.9 & 0.12 & 2.8 \\
$B_{S}^{0}$ & 5369.2 & 5367.4 & 0.40 & 0.9 & 5365.0 & 5371.3 & .007 & 3.2 \\
\hline
\end{tabular}

a. function of $p_{T}$ of either muon and eliminates the difference between $B^{+}$and $B^{-}$. The effect on the mean $B$ mass is minimal. However, because the effect is not well understood, we determine an uncertainty from the size of the correction weighted by the difference in $B^{-}$and $B^{+}$yields. No error is included for the charge-symmetric $\psi K^{*}$ and $\psi \phi$ modes.

- Polar Angle

Figure 4 shows a variation of $J / \psi$ mass as a function of the difference between the polar angle of the two muons, $\Delta \cot \theta$. To investigate the possible bias related to the polar angles of the tracks, we separate data in each mode into equal parts cutting on polar angle between the $J / \psi$ and the $K, K^{*}$ or $\phi$ and between vector daughters. Table 2 lists the fit $B$ masses in each case. For each $B$ meson, we use half of the larger mass difference as an estimate of the error. The effect is not understood and gives the dominant systematic uncertainty.

Table 3 summarizes the sources of systematic uncertainty in the mass measurement. The three $B$-meson masses are:

$$
\begin{aligned}
M\left(B^{+}\right) & =5279.6 \pm 1.7 \pm 2.9 \mathrm{MeV} / c^{2} \\
M\left(B^{0}\right) & =5279.9 \pm 2.5 \pm 3.7 \mathrm{MeV} / c^{2} \\
M\left(B_{S}^{0}\right) & =5367.7 \pm 2.4 \pm 4.8 \mathrm{MeV} / c^{2}
\end{aligned}
$$

where the first error is statistical and the second is systematic. Figure 5 shows the change in the mass for $B_{S}^{0}$ candidates in the two significant bins of figure 2 of reference 2 when analyzed with

Table 3: Summary the sources of systematic uncertainty in the $B$ meson mass measurements.

\begin{tabular}{|c|c|c|c|}
\hline Systematic Effect & $B^{+}$ & $B^{0}$ & $B_{S}^{0}$ \\
\hline$p_{T}$ Scale & 1.3 & 1.2 & 1.1 \\
Material Scale & 0.4 & 0.6 & 0.6 \\
Mass Fitting & 0.4 & 0.9 & 1.2 \\
Pointing Constraint & 0.2 & 0.2 & 0.2 \\
Covariance Matrix Scale & 1.0 & 1.0 & 1.0 \\
Selection Criteria & 1.5 & 1.5 & 2.8 \\
False Curvature & 0.7 & 0.0 & 0.0 \\
$\Delta \cot \theta$ & 1.0 & 2.8 & 3.2 \\
\hline Total $\left(\mathrm{MeV} / \mathrm{c}^{2}\right)$ & 2.9 & 3.7 & 4.8 \\
\hline
\end{tabular}




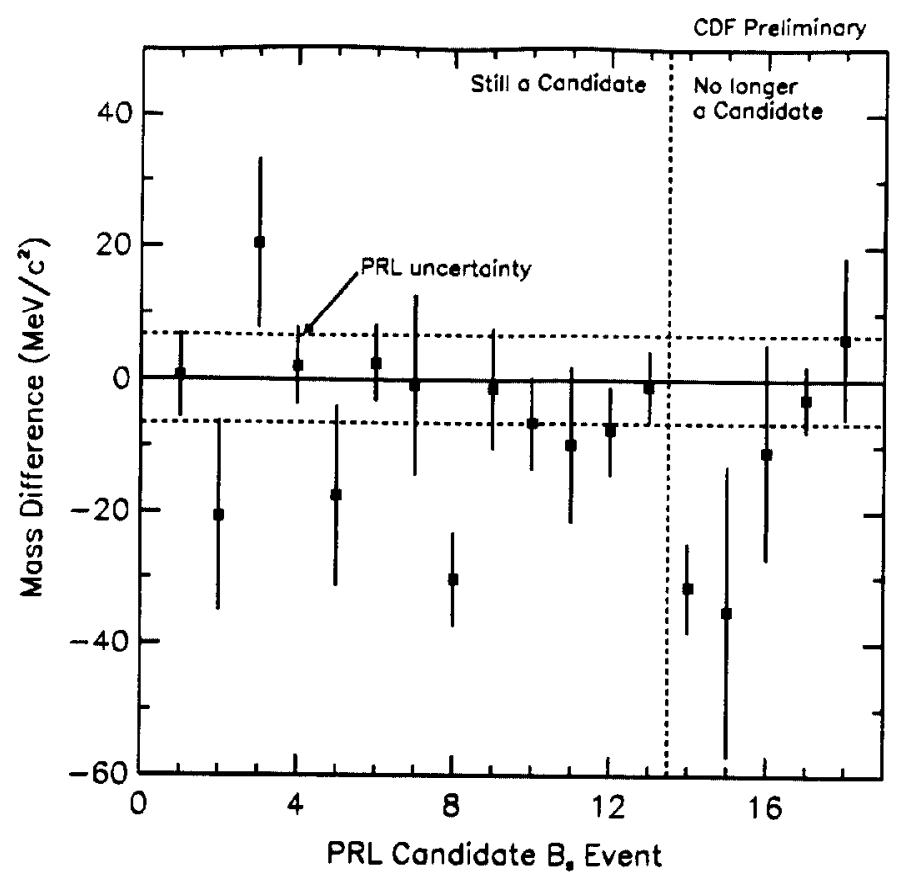

Figure 5: The change in reconstructed $B_{S}^{0}$ mass in the new analysis for events included in reference 2 .

the improved tracking chamber alignments. Several events now fail the selection cuts. However, the shift in mass is small for the events that are candidates in the earlier analysis and the one described here. Furthermore, the $B^{+}$and $B^{0}$ mass measurements are essentially unaffected. High-statistics comparisons of the two versions of track reconstruction using candidates from the $B$ mass sidebands with the cuts relaxed show event-by-event changes in the mass that are typically less than $3 \mathrm{MeV} / c^{2}$ in all three modes. Therefore, we believe the difference between the new measurement of $M\left(B_{S}^{0}\right)$ and the earlier published value ${ }^{2)}$ is consistent with a statistical fluctuation. The improved result is in good agreement with the result of ALEPH,) $M\left(B_{S}^{0}\right)=5368.6 \pm 5.6 \pm 1.5 \mathrm{MeV} / c^{2}$. The systematic uncertainty is currently dominated by the track $\cot \theta$ measurement. Study of this effect is ongoing. The values for the $B^{+}$and $B^{0}$ masses are in good agreement with the average ${ }^{10)}$ of CLEO and ARGUS results, $M\left(B^{+}\right)=$ $5278.7 \pm 0.2 \pm 2.0 \mathrm{MeV} / c^{2}$ and $M\left(B^{0}\right)=5278.9 \pm 0.2 \pm 2.0 \mathrm{MeV} / c^{2}$. The measurements from $B$ 's produced at the $\Upsilon(4 S)$ resonance are limited by knowledge of the beam energy. With improved understanding biases in track reconstruction and the addition of data from Run 1B, CDF $B$-mass measurements will be quite precise, and results for $B^{+}$and $B^{0}$ can be competitive with those from CLEO with an independent set of systematic uncertainties.

\section{$3 \quad B$ Meson Lifetimes}

The measurement of individual $B$ meson lifetimes is a necessary component to determine the CKM matrix element $V_{c b}$ as well as the mixture of charged and neutral $B$ production at the $\Upsilon(4 \mathrm{~S})$ resonance. The difference between the lifetimes of $B^{+}$and $B^{0}$ mesons provides a measure of the contribution of non-spectator effects in $B$-meson decay. Recent predictions ${ }^{11)}$ of the 


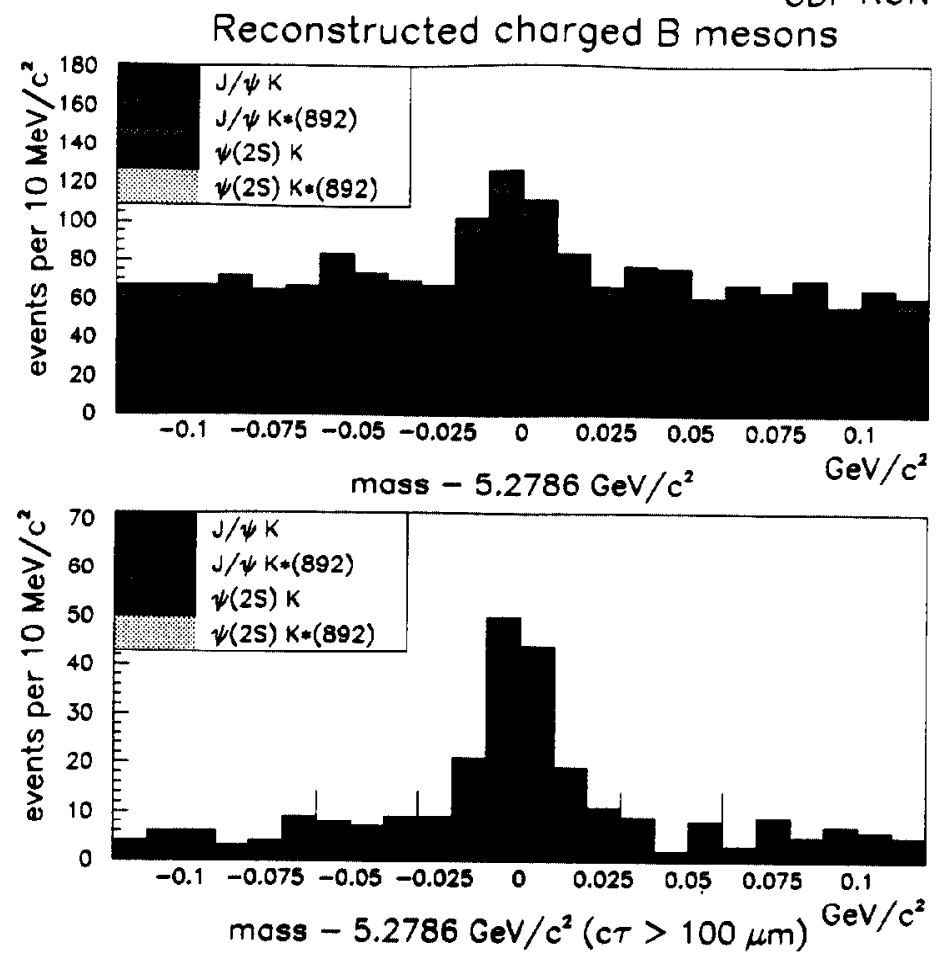

Figure 6: Mass distribution for all $B^{+}$candidates used in lifetime fit are shown by decay mode. The lower plots show the distributions after a proper lifetime cut $c \tau>100 \mu \mathrm{m}$, indicating substantial $B$ signals.

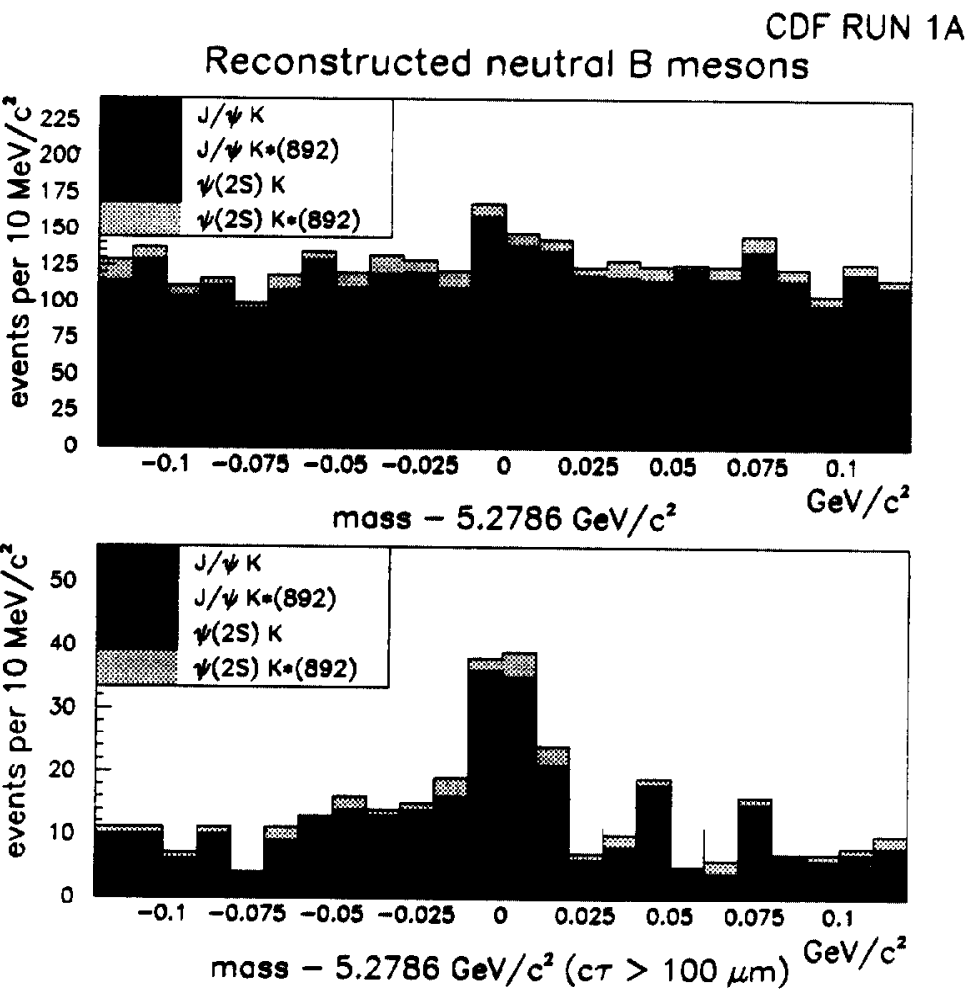

Figure 7: Mass distribution for all $B^{0}$ candidates used in lifetime fit are shown by decay mode. The lower plots show the distributions after a proper lifetime cut $c \tau>100 \mu \mathrm{m}$, indicating substantial $B$ signals. 
lifetimes find

$$
0.95 \tau\left(B^{+}\right) \lesssim \tau\left(B^{0}\right) \simeq \tau\left(B_{S}^{0}\right)
$$

To measure the separate lifetimes of $B^{0}$ and $B^{+}$mesons, we fully reconstruct their decays into modes containing a two muons and charged hadrons in the final state:

$$
\begin{array}{ll}
B^{+} \rightarrow J / \psi K^{+} & B^{0} \rightarrow J / \psi K_{S}^{0} \\
B^{+} \rightarrow J / \psi K^{*+} & B^{0} \rightarrow J / \psi K^{* 0} \\
B^{+} \rightarrow \psi^{\prime} K^{+} & B^{0} \rightarrow \psi^{\prime} K_{S}^{0} \\
B^{+} \rightarrow \psi^{\prime} K^{*+} & B^{0} \rightarrow \psi^{\prime} K^{* 0}
\end{array}
$$

where $J / \psi \rightarrow \mu^{+} \mu^{-}, \psi^{\prime} \rightarrow J / \psi \pi^{+} \pi^{-}, K^{* 0} \rightarrow K^{+} \pi^{-}, K^{*+} \rightarrow K_{S}^{0} \pi^{+}$, and $K^{*} \rightarrow \pi^{+} \pi^{-}$. Muons are selected with the same requirements as in the mass analysis. Because the impact parameter resolution of the SVX is critical to the lifetime measurement, we require that at least the two muon tracks be reconstructed in the SVX. As in the mass analysis, any track that is not part of the dimuon candidate can be assigned the pion or kaon hypothesis and used in reconstruction of any of the decay modes studied. $K_{S}^{0}$ candidates are formed from oppositely charged tracks with impact parameters greater than $2 \sigma$, where $\sigma$ is the combination of error of the track reconstruction and the beam profile width. A $K_{S}^{0}$ candidate's decay vertex must lie forward of the intersection of the two muon tracks, and the impact parameter of the $K_{S}^{0}$ candidate with respect to the $J / \psi$ vertex must be less than $2 \mathrm{~mm}$. We require mass of a $J / \psi$ candidate to be within $80 \mathrm{MeV} / c^{2}$ of the world average ${ }^{8)}$ mass; $\psi^{\prime}$, within $20 \mathrm{MeV} / c^{2} ; K^{*}$, within $80 \mathrm{MeV} / c^{2}$; and $K_{S}^{0}$, within $20 \mathrm{MeV} / c^{2}$. To reduce combinatorial background, we require $p_{T}(B)>6 \mathrm{GeV} / c$, and $p_{T}\left(K / K^{*}\right)>1.25 \mathrm{GeV} / c$.

We calculate the $B$ candidate mass and $p_{T}$ subject to the constraints that all the tracks or $K_{S}^{0}$ candidates come from a single point and that the $J / \psi, \psi^{\prime}$ and $K_{S}^{0}$ candidates have the world-average mass.) The $\chi^{2}$ confidence level of the reconstruction must be greater than $1 \%$. If both $K \pi$ charge assignments yield a $B$ candidate with $\Delta M=\left|M_{B}-5278.6\right|<120 \mathrm{MeV} / c^{2}$, the choice is made as described in Section 2. If there remain two or more candidates in an event with $\Delta M<120 \mathrm{MeV} / c^{2}$, we choose the one with the greatest confidence level. Figures 6 and 7 show the reconstructed mass distributions for charged and neutral $B$ candidates.

For the measurement of the $B$ lifetimes, we define the peak region as $\Delta M<30 \mathrm{MeV} / c^{2}$, and the sideband as $60<\Delta M<120 \mathrm{MeV} / c^{2}$. We estimate the number of signal (background) events in the peak region to be $148 \pm 16$ for $B^{+}$and $121 \pm 16$ for $B^{0}$. The $B$ lifetimes are are determined in an unbinned fit of the decay length $c \tau=L_{x y} M_{B} / p_{T}$, where $L_{x y}$ is the distance from the decay vertex to the run-average beam position in the transverse plane. The fit includes the distributions from both the peak and sideband regions. The peak region contains both signal and background events. We assume that the $c \tau$ distribution for events in the sideband region is the same as for the background in the peak region. The measured decay length of signal events is distributed according to an exponential (with slope $\lambda$ ) convoluted with a Gaussian resolution function. The resolution computed from the reconstruction for each event is used in the lifetime fit. The shape of the background distribution is similar to that used in the inclusive lifetime measurement.) A component presumed to be random combinations of prompt $J / \psi$ 's with other prompt tracks is described by the resolution function at the origin. $J / \psi$ 's from $B$ decays can be combined with random additional tracks including some from either $B$ in the event. These combinations contribute to the $c \tau$ distribution with an apparent lifetime. Because the particles that form the candidate may be taken from any part of an event, the displacement of the decay vertex may be opposite from the momentum direction yielding an apparent negative lifetime. 
Table 4: Results of the lifetime fits.

\begin{tabular}{|ll|rrr|rrr|}
\hline \multicolumn{2}{|c|}{ Parameter } & \multicolumn{3}{|c|}{$B^{+}$} & \multicolumn{3}{|c|}{$B^{0}$} \\
\hline$\lambda$ & {$[\mu \mathrm{m}]$} & 482 & \pm & 48 & 472 & \pm & 55 \\
$\alpha$ & {$[\%]$} & 26.9 & \pm & 2.3 & 14.4 & \pm & 1.8 \\
$\mathcal{N}$ & {$[$ events $]$} & 148 & \pm & 16 & 121 & \pm & 16 \\
$f^{-}$ & {$[\%]$} & 2.3 & \pm & 0.6 & 1.3 & \pm & 0.3 \\
$\lambda^{-}$ & {$[\mu \mathrm{m}]$} & 349 & \pm & 79 & 514 & \pm & 114 \\
$f^{+}$ & {$[\%]$} & 10.2 & \pm & 1.4 & 11.0 & \pm & 1.2 \\
$\lambda^{+}$ & {$[\mu \mathrm{m}]$} & 281 & \pm & 37 & 191 & \pm & 19 \\
\hline \multicolumn{2}{|c|}{ Confidence level } & \multicolumn{3}{|c|}{$43 \%$} & & $32 \%$ \\
\hline
\end{tabular}

These contributions to the background are described by exponentials over positive and negative $c \tau$ with slopes $\lambda^{+}$and $\lambda^{-}$and with weights $f^{+}$and $f^{-}$. The ratio of the number of background events in the peak region compared to the sideband region $(\alpha)$ is allowed to float in the fits within Poisson errors. The $c \tau$ distributions for peak and sideband events for $B^{0}$ and $B^{+}$are shown with the results of the lifetime fit in figures 8 and 9. Table 4 lists the results of the lifetime fit with statistical uncertainties only.

We have performed several consistency checks on the data including fitting $B$ and $\bar{B}$ separately, fitting early and later parts of the run separately, using the $J / \psi$ vertex as the $B$ vertex, requiring all tracks to be reconstructed in the SVX, and tighter kinematic cuts. All the tests give results consistent with the results of the standard fit within the statistical uncertainties. We have evaluated several sources of systematic uncertainties, estimating the uncertainty from the change in the lifetimes when various parts of the reconstruction or fit are altered.

- Residual Misalignment

Position offsets for each SVX wedge are found with electrons from $W$ events. The impact parameters of tracks used in the $B$ lifetime fits are altered by these offsets, and the variation in the measured lifetimes provides an estimate of the uncertainty.

- Trigger Bias

The CFT track processor assumes tracks originate at geometric center of the CTC. The effect on the lifetime of the dependence of CFT efficiency on track impact parameter is determined in Monte Carlo studies.

- Beam Stability

We measure the deviation of the average beam position over the duration of several typical Tevatron stores and take the variation to be an estimate of the movement of the beam position within a store.

- Resolution Scale

In order to account for the limitations in our understanding of the reconstruction resolution, we perform a new fit with an additional parameter that multiplies event-by-event resolution.

- Tails in Resolution Function

The distribution of average resolution for all events has long tails. We fit the lifetime with an event-by-event resolution function that includes these tails. 

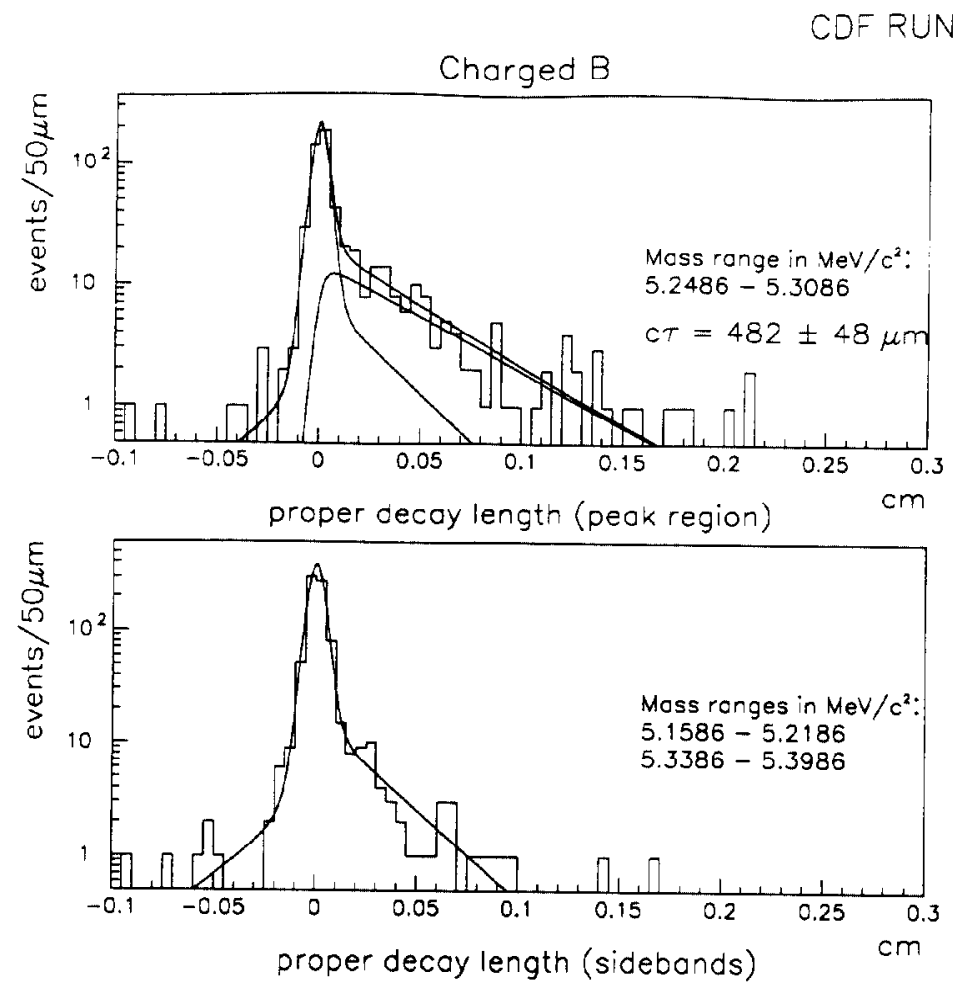

Figure 8: Lifetime distributions for the candidates in the peak region of figure 6 . The solid curves indicate the results of the lifetime fit for the signal and background functions and their sum.
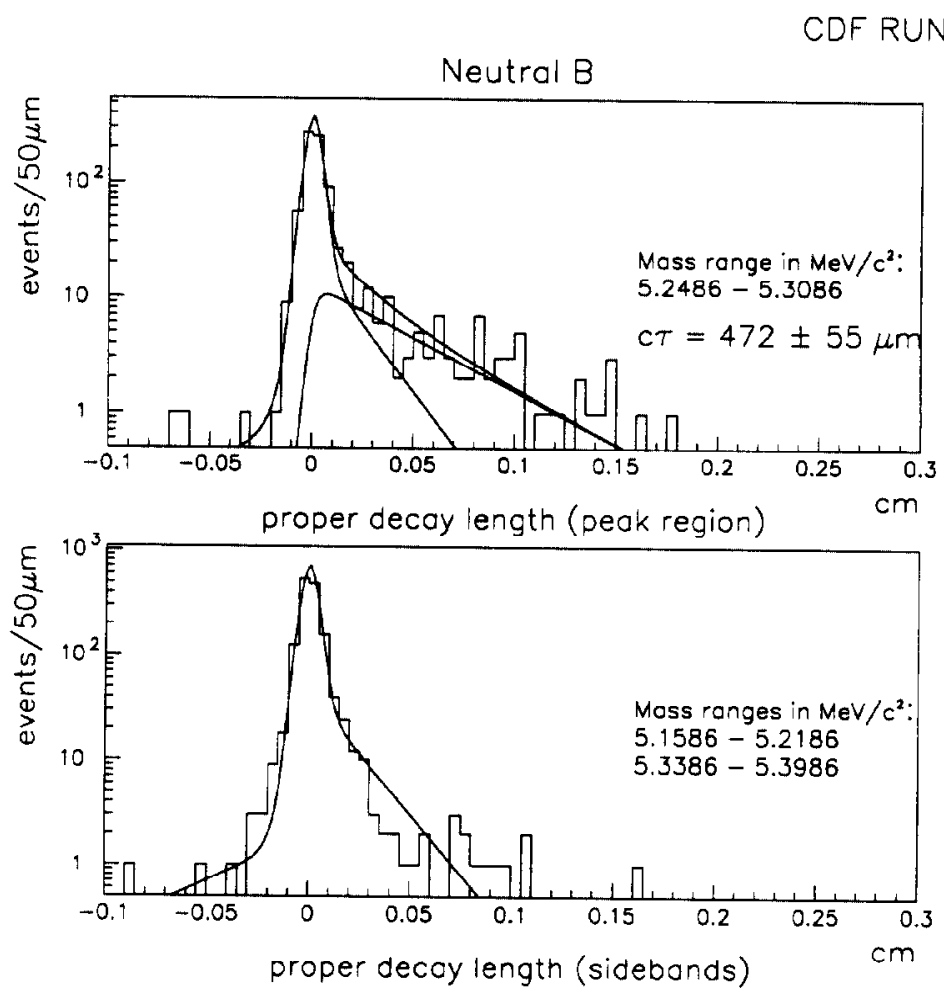

Figure 9: Lifetime distributions for the candidates in the peak region of figure 7 with the fit results superimposed as above. 


\begin{tabular}{|l|r|r|}
\hline Source of systematic error & Error on $B^{+}$ & Error on $B^{0}$ \\
\hline Residual misalignment & $10 \mu \mathrm{m}$ & $10 \mu \mathrm{m}$ \\
Trigger bias & $6 \mu \mathrm{m}$ & $6 \mu \mathrm{m}$ \\
Beam stability & $5 \mu \mathrm{m}$ & $5 \mu \mathrm{m}$ \\
Scale factor for resolution & $1 \mu \mathrm{m}$ & $2 \mu \mathrm{m}$ \\
Non-gaussian tails in resolution function & $7 \mu \mathrm{m}$ & $4 \mu \mathrm{m}$ \\
Fitting procedure bias & $2 \mu \mathrm{m}$ & $2 \mu \mathrm{m}$ \\
Background parameterization & $6 \mu \mathrm{m}$ & $21 \mu \mathrm{m}$ \\
\hline Total & $16 \mu \mathrm{m}$ & $25 \mu \mathrm{m}$ \\
\hline
\end{tabular}

Table 5: Summary of systematic uncertainties in lifetime measurements.

- Background Shape

We add a constant level to the background functions extending over the range $0<c \tau<$ $2 \mathrm{~mm}$ and refit.

- Fitting Procedure

To check for a bias, we fit many Monte Carlo samples with the number of observed signal and background events generated with measured parameters.

Table 5 summarizes the contributions to the systematic uncertainty. We find the separate lifetimes to be:

$$
\begin{gathered}
\tau\left(B^{+}\right)=1.61 \pm 0.16 \pm 0.05 \quad \text { ps } \\
\tau\left(B^{0}\right)=1.57 \pm 0.18 \pm 0.08 \text { ps }
\end{gathered}
$$

yielding a lifetime ratio

$$
\tau\left(B^{+}\right) / \tau\left(B^{0}\right)=1.02 \pm 0.16 \pm 0.05 \text {. }
$$

These results can be compared to the average from those from the LEP experiments ${ }^{12)}$ of $\tau\left(B^{+}\right)=1.66 \pm 0.14 \mathrm{ps}, \tau\left(B^{0}\right)=1.51 \pm 0.12 \mathrm{ps}$ and $\tau\left(B^{+}\right) / \tau\left(B^{0}\right)=1.10 \pm 0.14$. These results are consistent with small contributions from non-spectator decay processes.

\section{Conclusions}

Exclusive reconstruction of $B$ mesons has proven to be a fruitful tool for exploring heavy-quark physics at the Tevatron. Because all the measurements are limited by statistical uncertainties the expected fourfold addition to the data sample from the run in progress will provide increased precision to the understanding of the structure and decay of $B$ mesons. 


\section{References}

[1] F. Abe, et al., Phys. Rev. Lett. 68, 3403 (1992); F. Abe, et al., Phys. Rev. Lett. 69, 3704 (1992); F. Abe, et al., Phys. Rev. D47, 2639 (1993); F. Abe, et al., Phys. Rev. Lett. 71, 2537 (1993).

[2] F Abe, et al., Phys. Rev. Lett. 71, 1685 (1993).

[3] F. Abe, et al., Phys. Rev. Lett. 71, 3421 (1993).

[4] F. Abe, et al., Nucl. Inst. Meth. A271, 387 (1988).

[5] F. Bedeschi, et al., Nucl. Inst. Meth. A268, 50 (1988).

[6] G.W. Foster, et al., Nucl. Inst. Meth. A269, 93 (1988).

[7] A. Duncan, et al., "Masses and Decay Constants of Heavy-Light Mesons Using the Multistate Smearing Technique," FERMILAB-CONF-93/376-T; W. Kwong and J. Rosner, Phys. Rev. D44, 212 (1991); A. Martin, in Heavy Flavors and High Energy Collisions in the 1-100 TeV Range, A. Ali and L Cifarelli ed., 141, Plenum, New York (1989); S. Godfrey and N. Isgur, Phys. Rev. D32, 189 (1985).

[8] Particle Data Group, Phys. Rev. D45, S1 (1992).

[9] D. Buskulic, et al., Phys. Lett. B311, 425 (1993).

[10] T.E. Browder, K. Honsheid, and S. Playfer, "A Review of Hadronic and Rare B Decays," CLNS-93-1261. To appear in $B$ Decays, $2^{\text {nd }}$ edition, S. Stone ed., World Scientific: Singapore.

[11] I. Bigi, et al."Nonleptonic Decays of Beauty Hadrons: From Phenomenology to Theory," CERN-TH-7132-94. To appear in $B$ Decays, $2^{\text {nd }}$ edition, S. Stone ed., World Scientific: Singapore.

[12] P. Abreu, et al., Z. Phys. C57, 181 (1993); D. Buskulic, et al., Phys. Lett. B287, 449 (1992); D. Buskulic, et al., Phys. Lett. B307, 194 (1993); D. Buskulic, et al., Phys. Lett. B314, 459 (1993); P.D. Acton, et al., Phys. Lett. B307, 247 (1993). 